\title{
Quantum kinetic equation and universal conductance fluctuations in graphene
}

\author{
K. Kechedzhi, ${ }^{1}$ O. Kashuba, ${ }^{1}$ and Vladimir I. Fal'ko ${ }^{1,2}$ \\ ${ }^{1}$ Department of Physics, Lancaster University, Lancaster LA1 4YB, United Kingdom \\ ${ }^{2}$ Laboratoire de Physique des Solides, Universite Paris-Sud, CNRS UMR 8502, F-91405 Orsay, France
}

(Received 15 January 2008; revised manuscript received 8 April 2008; published 14 May 2008)

\begin{abstract}
We analyze universal conductance fluctuations (UCFs) in graphene in the framework of diagrammatic perturbation theory in the metallic regime. It is shown that strong intervalley scattering lifts the valley degeneracy of electronic states, whereas at weak intervalley scattering two valleys independently contribute such that the variance of UCF would be expected to show sample- and geometry-dependent behaviors.
\end{abstract}

DOI: 10.1103/PhysRevB.77.193403

PACS number(s): 73.23.-b, 73.43.Qt, 74.78.Na, 81.05.Uw

The unusual chiral properties of charge carriers in graphene $^{1-4}$ have recently received a lot of attention. Several theories have been developed ${ }^{5-9}$ interpreting observation of quantum interference effects in graphene, such as weak localization magnetoresistance ${ }^{10,11}$ and the Josephson proximity effect in superconductor-graphene-superconductor junctions. ${ }^{12}$ Low-temperature magnetoresistance measurements ${ }^{10,13,14}$ have shown universal conductance fluctuations (UCFs) which appear to be robust over a wide range of electron concentrations and magnetic fields, and numerical simulations of transport in monolayer graphene with charged disorder showed sample-to-sample variation of conductance. ${ }^{15}$ In this Brief Report, we analyze UCF using the same framework as the earlier weak localization studies. ${ }^{5,7}$ Specifically, we study UCF in graphene with various types of disorder in the fully developed metallic regime $\left(k_{F} l \gg 1\right)$, using quantum kinetic equation for diffusive transport in graphene and technique of semiclassical Keldysh functions.

The transport in graphene is determined by the lowenergy properties of charge carriers in the vicinity of corners ( $K$ points) of hexagonal Brillouin zone, ${ }^{16}$ called valleys. In the case of monolayer graphene, this can be described using the Hamiltonian, ${ }^{17,18}$

$$
\begin{gathered}
\hat{H}=v \vec{\Sigma} \mathbf{p}+\hat{h}_{w}(\mathbf{p})+\hat{V}(\mathbf{r}), \\
\hat{h}_{w}=-\mu \Sigma_{x}(\vec{\Sigma} \mathbf{p}) \Lambda_{z} \Sigma_{x}(\vec{\Sigma} \mathbf{p}) \Sigma_{x} .
\end{gathered}
$$

Here, the basis of bispinors $\Phi=\left[\phi_{\mathbf{K}_{+}, A}, \phi_{\mathbf{K}_{+}, B}, \phi_{\mathbf{K}_{-}, B}, \phi_{\mathbf{K}_{-}, A}\right]$ characterizes electronic amplitudes on two crystalline sublattices of graphene $(A$ and $B) . \Sigma_{s}$ and $\Lambda_{l},(l=x, y, z)$ are $4 \times 4$ matrices in the valley and sublattice spaces, ${ }^{19,20}$ introduced in Ref. 5. The momentum $\mathbf{p}=p(\cos \varphi, \sin \varphi)$ is defined with respect to the $\mathbf{K}$ points. ${ }^{16}$ The first, "Dirac" term in Eq. (1) determines an almost linear spectrum $\epsilon= \pm v p$ of electrons. The trigonal warping term, $\hat{h}_{w}$, takes into account a slight trigonal asymmetry of the Fermi line of graphene in one valley $[$ such that $\epsilon(\mathbf{K}, \mathbf{p}) \neq \boldsymbol{\epsilon}(\mathbf{K},-\mathbf{p})]$, which will be treated below as a weak perturbation. Due to the time-reversal symmetry of the system, the trigonal warping has opposite sign in $\mathbf{K}$ and $\mathbf{K}^{\prime}$ valleys, $\epsilon(\mathbf{K}, \mathbf{p})=\epsilon\left(\mathbf{K}^{\prime},-\mathbf{p}\right)$, which is taken into account by the valley matrix structure of $\hat{H}$. The timereversal-symmetric disorder has the form ${ }^{20}$

$$
\hat{V}(\mathbf{r})=u(\mathbf{r})+\sum \Sigma_{s} \Lambda_{l} u_{s l}(\mathbf{r}) .
$$

The first term in $\hat{V}(\mathbf{r})$ (a unit matrix in the valley and sublattice space) takes into account Coulomb potential of remote charges in, or on the surface of, $\mathrm{SiO}_{2}$ substrate. Valleydiagonal part of $\hat{V}(\mathbf{r}), \Sigma_{x / y} \Lambda_{z} u_{x / y, z}(\mathbf{r})$, takes into account symmetry-breaking disorder smooth on the scale of lattice constant such as a gradual variation of $A-B$ bond length, whereas $\Sigma_{z} \Lambda_{z} u_{z, z}(\mathbf{r})$ accounts for different on-site potentials on $A$ and $B$ sublattices. Atomically sharp potentials from lattice defects or a dopant directly deposited on graphene can scatter with a large momentum transfer $\sim \hbar / a$ ( $a$ is a lattice constant) and therefore mix two valley states of electrons. Such defects are taken into account by valley-off-diagonal part of $\hat{V}(\mathbf{r})$. The disorder is characterized by correlators $\left\langle u_{s l}(\mathbf{r}) u_{s^{\prime} l^{\prime}}\left(\mathbf{r}^{\prime}\right)\right\rangle=\delta\left(\mathbf{r}-\mathbf{r}^{\prime}\right) \delta_{s s^{\prime}} \delta_{l l^{\prime}} w_{s l}$, and this determines the corresponding scattering rates $\tau_{s l}^{-1}=\delta_{s, s^{\prime}} \delta_{l, l^{\prime}} \pi \gamma w_{s, l} / \hbar$ [where $\gamma=k_{F} /(2 \pi v \hbar)$ is the Fermi density of states in doped graphene]. After averaging over impurity configurations, the scattering rates should preserve rotational symmetry of graphene which means that $\tau_{x l}=\tau_{y l} \equiv \tau_{\perp l}$ and $\tau_{s x}=\tau_{s y} \equiv \tau_{s \perp}$. Two scattering rates, $\tau_{\mathrm{z}}^{-1}=4 \tau_{\perp z}^{-1}+2 \tau_{z z}^{-1}$ and $\tau_{\mathrm{i}}^{-1}=4 \tau_{\perp \perp}^{-1}+2 \tau_{z \perp}^{-1}$, describe the valley-diagonal and valley off-diagonal parts of the symmetry-breaking disorder potential, respectively, whereas the total scattering rate is $\tau^{-1}$ $=\tau_{0}^{-1}+\tau_{z z}^{-1}+2 \tau_{\perp z}^{-1}+2 \tau_{z \perp}^{-1}+4 \tau_{\perp \perp}^{-1}$.

To characterize the UCF, we evaluate the variance of conductance, $\left\langle\delta \mathcal{G}^{2}\right\rangle=\left\langle\mathcal{G}^{2}\right\rangle-\langle\mathcal{G}\rangle^{2}$, where the angular brackets stand for averaging with respect to disorder configurations. The main order of $\left\langle\delta \mathcal{G}^{2}\right\rangle$ in $1 / k_{F} l \ll 1$ is given by perturbation theory diagrams shown in Figs. 1(a) and 1(b). ${ }^{21}$ These diagrams consist of Hikami boxes [shaded blocks in Figs. 1(a) and $1(\mathrm{~b})$ ] connected by the wavy lines, which represent the sum of ladder diagrams: diffusons and Cooperons. ${ }^{21}$ Cooperons are strongly suppressed in magnetic fields in which magnetic flux is larger than flux quantum per sample area. Since the UCF are usually experimentally studied in such a high magnetic field regime, here we neglect the contribution of the Cooperon diagrams. In contrast, diffusons, which are Green functions of quantum diffusion equation, are not suppressed by a magnetic field although as we will show below their contribution depends on the efficiency of the symmetrybreaking disorder in the system. Below, we obtain diffusons by analyzing quantum kinetic equation in disordered graphene using the semiclassical approximation $\left(k_{F} l \gg 1\right)$. 
(a) $\mathrm{R}$

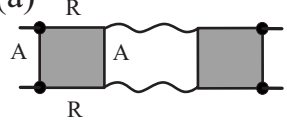

(b)

(c)

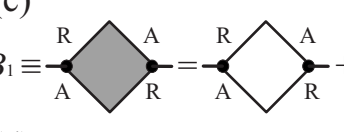

(d)
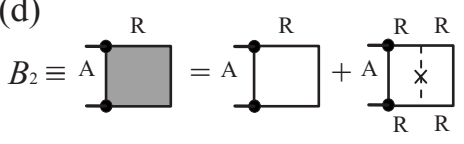

(e)



FIG. 1. [(a) and (b)] The diagrams which contribute to the main order in the diagrammatic expansion of the conductivityconductivity correlation function. Here, the solid lines stand for the impurity averaged retarded or advanced Green functions, the filled circles stand for the renormalized current vertices and the long wavy lines stand for the diffusion ladders. [(c) and (d)] Hikami boxes of two types and additional diagrams which determine renormalization in the main order in $1 / k_{F} l \ll 1$. The dashed lines correspond to the disorder potential. (e) Diagrammatic equation for renormalized current vertex.

Quantum kinetic equation describes relaxation of nonequilibrium inhomogeneous distribution of electrons in a disordered system. Using matrix Green functions in Keldysh representation, $\check{G}\left(\mathbf{r}_{1}, t_{1}, \mathbf{r}_{1^{\prime}}, t_{1^{\prime}}\right)$, we derive semiclassical form of Green functions for electrons in graphene. We separate slow and fast variables in $\check{G}$ : $\mathbf{r}=\frac{1}{2}\left(\mathbf{r}_{1}+\mathbf{r}_{1^{\prime}}\right)$ and $t=\frac{1}{2}\left(t_{1}\right.$ $\left.+t_{1^{\prime}}\right)$, which vary at $r \gg 1 / k_{F}$ and $t \gg \hbar / \boldsymbol{\epsilon}_{F}$, and $\delta \mathbf{r}=\mathbf{r}_{1}-\mathbf{r}_{1^{\prime}}$ and $\delta t=\left(t_{1}-t_{1^{\prime}}\right)$, which vary at $\delta r \sim 1 / k_{F}$ and $\delta t \sim \hbar / \epsilon_{F}$. We then take the Fourier transform of $\check{G}$ with respect to $\delta t$ and $\delta \mathbf{r}$. The Fourier-transformed Green function,

$$
\check{G}(\epsilon, \mathbf{p}, \mathbf{r}, t)=\left(\begin{array}{cc}
\hat{G}^{R}(\epsilon, \mathbf{p}, \mathbf{r}, t) & \hat{G}^{K}(\epsilon, \mathbf{p}, \mathbf{r}, t) \\
0 & \hat{G}^{A}(\epsilon, \mathbf{p}, \mathbf{r}, t)
\end{array}\right),
$$

obeys the following Dyson's equation: ${ }^{20}$

$$
\begin{aligned}
& \left(\frac{i}{2} \partial_{t}+\frac{i}{2} v \Sigma \nabla_{\mathbf{r}}-\hat{h}_{w}+\epsilon-v \Sigma \mathbf{p}-\check{S}\right) \check{G}=1, \\
& \check{S} \equiv\left(\begin{array}{cc}
\hat{S}^{R} & \hat{S}^{K} \\
0 & \hat{S}^{A}
\end{array}\right)=\int \frac{d^{2} \mathbf{p}}{(2 \pi)^{2}}\langle\hat{V} \check{G}(\mathbf{p}, \epsilon, \mathbf{r}, t) \hat{V}\rangle,
\end{aligned}
$$

where in the self-energy $\check{S}, \hat{S}^{R / A}=\mp i / 2 \tau$ (Ref. 20) for $\epsilon_{F} \tau$ $\gg 1$. In the following derivation, Eqs. (4)-(20), we put $\hbar=1$, and restore the Planck's constant in the final answers.

The semiclassical Green function which describes lowenergy properties of the system is defined as ${ }^{20}$

$$
\check{g}(\epsilon, \mathbf{n}, \mathbf{r}, t)=\frac{i}{\pi} \int d \xi \check{G}[\epsilon, \mathbf{n}(\epsilon+\xi), \mathbf{r}, t],
$$

where integration over $\xi$ is performed in the vicinity of the Fermi level. ${ }^{22}$ The response of the system to external pertur- bation is described by occupation numbers determined by the Keldysh component of semiclassical Green function. An equation for the latter can be derived using the gradient expansion, that is, assuming that $\check{G} \gg l \nabla_{\mathbf{r}} \check{G}, \tau \partial_{t} \check{G}$. Advanced and retarded components of $\breve{G}$ are taken into account in the zeroth order of the gradient expansion,

$$
\hat{G}^{R / A}=\frac{\epsilon+v \Sigma \mathbf{p}}{\left(\epsilon \pm \frac{i}{2 \tau}\right)^{2}-(v p)^{2}} .
$$

This determines the semiclassical Green functions ${ }^{20}$

$$
\hat{g}^{R / A}= \pm \frac{1}{2}(1+\Sigma \mathbf{n}),
$$

where $\mathbf{n}=\mathbf{p} / p$. Dyson's equation (4) for Keldysh component of disorder-averaged semiclassical Green function reads

$$
\begin{aligned}
\frac{i}{2} \partial_{t} \hat{g}^{K} & +\frac{i}{2} v \Sigma \nabla_{\mathbf{r}} \hat{g}^{K}+\epsilon \Sigma_{z}(1+\Sigma \mathbf{n}) \Sigma_{z} \hat{g}^{K}-\hat{h}_{w} \hat{g}^{K}+\frac{i}{2 \tau} \hat{g}^{K} \\
+ & \frac{1}{2} \hat{S}^{K}(1+\Sigma \mathbf{n})=0,
\end{aligned}
$$

where

$$
\hat{S}^{K}=-i \pi \gamma \int \frac{d \theta}{2 \pi}\left\langle\hat{V} \hat{g}^{K} \hat{V}\right\rangle .
$$

Note that, in Eq. (9), the energy $\epsilon$ is defined with respect to mass surface shifted due to the effects of disorder and Fermi line warping. ${ }^{23}$

Analyzing the main term in Eq. (9), $\epsilon \Sigma_{z}\left(1+\sum \mathbf{n}\right) \Sigma_{z} \hat{g}^{K}$, we find that the leading contribution to $\hat{g}^{K}$ is proportional to the matrix $(1+\Sigma \mathbf{n})$. Using ${ }^{20}$

$$
\hat{g}^{K}=\sum_{l=0, x, y, z}\left[g^{l}(1+\Sigma \mathbf{n})+\delta \hat{g}_{z}^{l}\right] \Lambda_{l},
$$

where $g^{l}$ are functions of $\mathbf{n}$ and $l=0, x, y, z$, we considered $\delta \hat{g}_{z}^{l}$ as a small correction (with an arbitrary matrix form ${ }^{24}$ ) and checked that the latter can be neglected in the leading order of the gradient expansion.

Kinetic equation is obtained from Eq. (9) by subtracting its Hermitian conjugate. After substituting the self-energies and $\hat{g}^{K}$ in the form (11), we find that

$$
\begin{aligned}
\partial_{t} g^{l} & +v \mathbf{n} \nabla g^{l}+\frac{1}{\tau}\left(g^{l}-\left\langle g^{l}\right\rangle_{\varphi}-\left\langle g^{l} \mathbf{n}^{\prime}\right\rangle_{\varphi} \mathbf{n}\right)+\delta^{l}\left\langle g^{l}\right\rangle_{\varphi} \\
& +\eta^{l}\left\langle g^{l} \mathbf{n}^{\prime}\right\rangle_{\varphi} \mathbf{n}+\sum \Upsilon_{l l^{\prime}} g^{l^{\prime}}=0 .
\end{aligned}
$$

The angular brackets $\langle\cdots\rangle_{\varphi}$ in Eq. (12) and below denote averaging over momentum directions, and the coefficients $\eta$ and $\delta^{d}$ are defined as

$$
\begin{gathered}
\delta^{0}=0, \quad \delta^{z}=8 \tau_{\perp \perp}^{-1}+4 \tau_{z \perp}^{-1}, \\
\delta^{x}=\delta^{y}=4 \tau_{\perp \perp}^{-1}+4 \tau_{\perp z}^{-1}+2 \tau_{z \perp}^{-1}+2 \tau_{z z}^{-1}, \\
\eta^{0}=4 \tau_{\perp \perp}^{-1}+2 \tau_{\perp z}^{-1}+4 \tau_{z \perp}^{-1}+2 \tau_{z z}^{-1},
\end{gathered}
$$




$$
\begin{gathered}
\eta^{z}=4 \tau_{\perp \perp}^{-1}+2 \tau_{\perp z}^{-1}+2 \tau_{z z}^{-1}, \\
\eta^{x}=\eta^{y}=4 \tau_{\perp \perp}^{-1}+2 \tau_{\perp z}^{-1}+2 \tau_{z \perp}^{-1} .
\end{gathered}
$$

The effect of the Fermi line asymmetry is taken into account by

$$
\Upsilon_{x y}=-\Upsilon_{y x}=\frac{v^{2}}{2 \mu \epsilon_{F}^{2}} n_{x}\left(1-4 n_{y}^{2}\right),
$$

whereas $\Upsilon_{l l^{\prime}}$ with $\left(l, l^{\prime}\right) \neq(x, y),(y, x)$ are equal to zero.

The gradient expansion of Eq. (12) leads to the diffusion equation for the angle-average density matrix $\left\langle g^{l}\right\rangle_{\varphi}$,

$$
\left[\partial_{t}+D_{l}(i \nabla)^{2}+\Gamma^{l}\right]\left\langle g^{l}\right\rangle_{\varphi}=0
$$

where $^{25}$

$$
D_{l}=v^{2} \tau_{t r}^{l} / 2, \quad \tau_{t r}^{l} \equiv 2 \tau /\left(1+\eta^{l} \tau\right)
$$

$\Gamma_{0}^{0}=0, \quad \Gamma_{0}^{z}=2 \tau_{i}^{-1}, \quad \Gamma_{0}^{x}=\Gamma_{0}^{y}=\tau_{w}^{-1}+\tau_{z}^{-1}+\tau_{i}^{-1} \equiv \tau_{*}^{-1}$.

Here, the valley-dependent transport times $\tau_{t r}^{l}$ are determined by the efficiency of backscattering of electrons in corresponding mixed valley states [described by density matrix components $\left.\left\langle g^{l}\right\rangle,(l=0, x, y, z)\right]$. Such that, for example, due to the chirality of electrons in graphene backscattering off the potential disorder is suppressed, ${ }^{5,18}$ and in a sample with purely potential disorder the transport time is given by $\tau_{t r}^{l}=2 \tau_{0} \quad(l=0, x, y, z)$. In realistic samples, symmetrybreaking disorder restores the backscattering, which results in reduced valley-state-dependent transport times $\tau_{t r}^{l}<2 \tau_{0}$. The relaxation gaps $\Gamma^{l}$ are induced in this picture by symmetry-breaking disorder and the Fermi line warping effect. This warping effect determines the different evolution operators of electrons in two different valleys and suppresses the intervalley coherence terms in the density matrix $\left\langle g^{x / y}\right\rangle$. At the same time, however, the intravalley components of density matrix $\left\langle g^{0 / z}\right\rangle$ are not affected by the trigonal warping. This effect is taken into account using a relaxation time,

$$
\tau_{w}^{-1}=2 \tau_{0}\left(\epsilon^{2} \mu / \hbar v^{2}\right)^{2}
$$

(we assumed $\tau_{w}^{-1} \ll \tau^{-1}$ ). Similarly, disorder terms $u_{z x / y} \Lambda_{z} \Sigma_{x / y}$ and $u_{z z} \Lambda_{z} \Sigma_{z}$ scatter electrons in different valley states differently, which also leads to relaxation of $\left\langle g^{x / y}\right\rangle$ without affecting $\left\langle g^{0 / z}\right\rangle$. Finally, intervalley disorder terms $u_{x s} \Lambda_{x} \Sigma_{s}$ and $u_{y s} \Lambda_{y} \Sigma_{s}(s=x, y, z)$ mix the two valley states and lead to relaxation of all "valley-triplet" components of density matrix $\left\langle g^{x / y / z}\right\rangle$, which is taken into account in Eq. (19) by the intervalley relaxation rate $\tau_{i}^{-1}$.

Diffusons, $\mathcal{D}^{l}$, can be now found as Green functions of diffusion equations (19) with initial inhomogeneous distribution $g_{0}^{l}$. To describe UCF in a small graphene sample, we solve diffuson equations (19) with boundary conditions at current contacts $\mathcal{D}^{l}=0$. The physical edge of graphene is atomically sharp and hence generates strong intervalley scattering, which suppresses valley-triplet diffuson modes near the edge, thus leading to the boundary condition, $\mathcal{D}^{x / y / z}=0$. In contrast, the particle-density ("singlet") mode $\mathcal{D}^{0}$ has boundary condition $(\mathbf{n} \nabla) \mathcal{D}^{0}=0$ corresponding to the absence of charge current through the edge. Solutions of the diffusion equations (19) for a rectangular graphene wire $L_{x} \times L_{y}$ are given by

$$
\begin{gathered}
\mathcal{D}^{l}\left(\mathbf{r}, \mathbf{r}^{\prime}\right)=\frac{1}{\pi \gamma \tau^{2}} \sum_{n=1}^{\infty} \sum_{m=0}^{\infty} \frac{\phi_{n, m}^{l}(\mathbf{r}) \phi_{n, m}^{l}\left(\mathbf{r}^{\prime}\right)}{D^{l} \pi^{2} \lambda_{n, m}^{l}}, \\
\phi_{n, m}^{0}(\mathbf{r})=\sqrt{\frac{2}{L_{x}} \frac{2}{L_{y}}} \sin \left(\frac{n \pi x}{L_{x}}\right) \cos \left(\frac{m \pi y}{L_{y}}\right), \\
\phi_{n, m}^{x / y / z}(\mathbf{r})=\sqrt{\frac{2}{L_{x}} \frac{2}{L_{y}}} \sin \left(\frac{n \pi x}{L_{x}}\right) \sin \left(\frac{m \pi y}{L_{y}}\right), \\
\lambda_{n, m}^{l}=\left(\frac{n^{2}}{L_{x}^{2}}+\frac{m^{2}}{L_{y}^{2}}\right)+\frac{\Gamma^{l}+\tau_{\varphi}^{-1}}{D^{l} \pi^{2}},
\end{gathered}
$$

where we take into account dephasing due to inelastic processes $\tau_{\varphi}$.

As compared to the conventional electrons systems, Hikami boxes $\hbar^{2} B_{1}^{l}=\frac{1}{2} e^{2} v_{0}^{2} \nu \tau^{2}\left(\tau_{t r}^{0}\right)^{2} / \tau_{t r}^{l}, \hbar^{2} B_{2}^{l}=\frac{1}{4} e^{2} v_{0}^{2} \nu \tau^{2}\left(\tau_{t r}^{0}\right)^{2} \tau_{t r}^{l}$, and the current vertex $\tilde{v}_{x}=v_{0} \tau_{t r}^{0} / \tau \Sigma_{x}$ in monolayer graphene have to be renormalized by additional diagrams shown in Figs. 1(c) and 1(d) and by vertex corrections (black dots in Fig. 1). Both of these corrections contribute to the variance of conductivity in the main order in $1 / k_{F} l$ and are nonvanishing since current operator in monolayer graphene is momentum independent. ${ }^{5}$

The variance of conductance fluctuations is a sum of diagrams shown in Figs. 1(a) and 1(b), in which the diagram of Fig. 1(a) is encountered twice in the diagrammatic expansion ${ }^{26}$ and hence has a combinatorial prefactor 2. As a result at $T=0$, we get $^{27}$

$$
\left\langle\delta \mathcal{G}^{2}\right\rangle=\frac{6}{L_{x}^{4}}\left(\frac{2 e^{2}}{h}\right)^{2} \sum_{l, n, m} \frac{C_{l}}{\left[\lambda_{n, m}^{l}\right]^{2}}, \quad C_{l}=\left(\frac{\tau_{t r}^{0}}{\tau_{t r}^{l}}\right)^{4} .
$$

It is interesting to compare UCF for rectangular phasecoherent graphene samples with $L_{x} \gg L_{y}$ and $L_{x} \ll L_{y}$. As mentioned before, we consider the system in an intermediate magnetic field. In a narrow wire, $\mathcal{D}^{x, y, z}$ decay at the length $\sim L_{y}$, and the variance of conductance is dominated by the valley-singlet diffuson component, $\mathcal{D}^{0}$, and, $\left\langle\delta \mathcal{G}^{2}\right\rangle$ $=\frac{1}{15}\left(2 e^{2} / h\right)^{2},{ }^{28}$ which coincides with the standard result for quasi-1D metallic wires in the unitary limit. ${ }^{21}$ In contrast, in the case of $L_{y} \gg L_{x}$, all diffuson components $\mathcal{D}^{l}, l=0, x, y, z$ may contribute to the variance, depending on whether the effect of trigonal warping induces suppression of intervalley diffuson components $\mathcal{D}^{x / y}$ or not. This determines $\left\langle\delta \mathcal{G}^{2}\right\rangle$ $=\alpha\left[3 \zeta(3) / 2 \pi^{3}\right]\left(L_{y} / L_{x}\right)\left(2 e^{2} / h\right)^{2} \quad[\zeta(n)$ is Riemann's zeta function] with $\alpha=4$ for $L_{x}<\sqrt{D_{0} \tau_{*}}, \alpha=C_{0}+C_{z} \approx 2$ for $\sqrt{D_{0} \tau_{*}}<L_{x}<\sqrt{D_{0} \tau_{i}}$, and $\alpha=1$ for $\sqrt{D_{0} \tau_{*, i}}<L_{x}$. Inelastic processes such as electron-electron or electron-phonon interactions limit the coherence length $L_{\varphi} \sim \sqrt{D_{0} \tau_{\varphi}}<L_{x / y}$ in the sample. In this case, conductance of the sample is determined as conductance of a network of resistors of size $L_{\varphi}$ each with conductance variance given by Eq. (26). Also, if at high temperatures $L_{T} \equiv \sqrt{\hbar D_{0} / k T}<L$ and $L_{T}<L_{\varphi}$ thermal broadening produces an additional self-averaging reducing the conductance fluctuations. For a long wire, 
$\left\langle\delta \mathcal{G}^{2}(T)\right\rangle \approx \pi / 3\left(2 e^{2} / h\right)^{2}\left(L_{T} / L_{x}\right)^{2}\left(L_{\varphi} / L_{x}\right)$, whereas for a square sample with dimensions $L_{x} \times L_{y}: \quad\left\langle\delta \mathcal{G}^{2}(T)\right\rangle$ $\approx \alpha / 3\left(2 e^{2} / h\right)^{2}\left(L_{y} / L_{x}\right)\left(L_{T} / L_{x}\right)^{2} \ln \left(L_{\varphi} / L_{T}\right)$.

In conclusion, we have shown that the variance of interference-induced conductance fluctuations in graphene is of the order of the usual UCF value in metals, with a prefactor dependent on the strength of the intervalley scattering and shape of the sample. In a long wire of graphene or in the material with strong intervalley scattering, the magnetofluctuations of conductance have the variance typical for the unitary symmetry class (intermediate magnetic field). In a wide graphene sample $\left(L_{x}<L_{y}\right)$ with weak intervalley scattering, the size of magnetoconductance fluctuations is increased as compared to unitary symmetry-class result by at least a factor $\sim 2$. This behavior is opposite to what was found for the weak localization magnetoresistance: ${ }^{7}$ the latter was sup- pressed in the case of weak intervalley scattering, whereas strong intervalley scattering was found to restore the weak localization effect. This behavior contrasts the observation that in usual metals with nonchiral electrons UCF scale similar to the weak localization correction to conductivity, made by Aleiner and Blanter. ${ }^{29}$ The analysis of the UCF in bilayer graphene showed a result very similar to the monolayer case despite a difference ${ }^{7,30}$ in the electronic spectrum.

The authors wish to thank E. McCann, B. Altshuler, A. Morpurgo, C. W. J. Beenakker, and A. Savchenko for helpful discussions. This project has been funded by EPSRC Grant No. EP/C511743 and ESF FoNE network SpiCo. They also thank K. Efetov for attracting our attention to that our UCF results for the intermediate asymptotic regimes coincide with those in Ref. 31.
${ }^{1}$ K. S. Novoselov et al., Nature (London) 438, 197 (2005); K. S. Novoselov et al., Science 306, 666 (2004); Y. Zhang et al., Phys. Rev. Lett. 94, 176803 (2005); Y. Zhang et al., Nature (London) 438, 201 (2005).

${ }^{2}$ K. S. Novoselov et al., Nat. Phys. 2, 177 (2006).

${ }^{3}$ A. H. Castro Neto et al., arXiv:0709.1163 (unpublished).

${ }^{4}$ C. W. J. Beenakker, arXiv:0710.3848 (unpublished).

${ }^{5}$ E. McCann et al., Phys. Rev. Lett. 97, 146805 (2006).

${ }^{6}$ I. L. Aleiner and K. B. Efetov, Phys. Rev. Lett. 97, 236801 (2006).

${ }^{7}$ K. Kechedzhi et al., Phys. Rev. Lett. 98, 176806 (2007).

${ }^{8}$ H. Suzuura and T. Ando, Phys. Rev. Lett. 89, 266603 (2002).

${ }^{9}$ M. Titov and C. W. J. Beenakker, Phys. Rev. B 74, 041401(R) (2006).

${ }^{10}$ S. V. Morozov et al., Phys. Rev. Lett. 97, 016801 (2006).

${ }^{11}$ R. V. Gorbachev et al., Phys. Rev. Lett. 98, 176805 (2007).

${ }^{12}$ H. B. Heersche et al., Nature (London) 446, 56 (2007).

${ }^{13}$ F. V. Tikhonenko et al., Phys. Rev. Lett. 100, 056802 (2008).

${ }^{14}$ R. V. Gorbachev et al., Physica E (Amsterdam) 40, 1360 (2008).

${ }^{15}$ A. Rycerz et al., Europhys. Lett. 79, 57003 (2007).

${ }^{16}$ Corners of the hexagonal Brillouin zone are $\mathbf{K}_{ \pm}= \pm\left(\frac{2}{3} h a^{-1}, 0\right)$ ( $a$ is the lattice constant).

${ }^{17}$ P. R. Wallace, Phys. Rev. 71, 622 (1947).

${ }^{18}$ T. Ando, J. Phys. Soc. Jpn. 74, 777 (2005).

${ }^{19}$ Isospin and pseudospin matrices are defined as

$$
\begin{aligned}
& \Sigma_{x}=\left[\begin{array}{cccc}
0 & 1 & 0 & 0 \\
1 & 0 & 0 & 0 \\
0 & 0 & 0 & -1 \\
0 & 0 & -1 & 0
\end{array}\right], \quad \Sigma_{y}=\left[\begin{array}{cccc}
0 & -i & 0 & 0 \\
i & 0 & 0 & 0 \\
0 & 0 & 0 & i \\
0 & 0 & -i & 0
\end{array}\right], \\
& \Sigma_{z}=\left[\begin{array}{cccc}
1 & 0 & 0 & 0 \\
0 & -1 & 0 & 0 \\
0 & 0 & 1 & 0 \\
0 & 0 & 0 & -1
\end{array}\right], \quad \Lambda_{x}=\left[\begin{array}{cccc}
0 & 0 & 1 & 0 \\
0 & 0 & 0 & -1 \\
1 & 0 & 0 & 0 \\
0 & -1 & 0 & 0
\end{array}\right] \text {, } \\
& \Lambda_{y}=\left[\begin{array}{cccc}
0 & 0 & -i & 0 \\
0 & 0 & 0 & i \\
i & 0 & 0 & 0 \\
0 & -i & 0 & 0
\end{array}\right], \quad \Lambda_{z}=\left[\begin{array}{cccc}
1 & 0 & 0 & 0 \\
0 & 1 & 0 & 0 \\
0 & 0 & -1 & 0 \\
0 & 0 & 0 & -1
\end{array}\right] \text {. }
\end{aligned}
$$

These matrices determine two commuting subgroups of the group $\mathrm{U}_{4}$ of unitary transformations of a four-component $\Phi$ : an isospin (sublattice) group $\mathrm{SU}_{2}^{\Sigma} \equiv\left\{e^{i a \vec{n} \cdot \vec{\Sigma}}\right\}$ and a pseudospin (valley) group $\mathrm{SU}_{2}^{\Lambda} \equiv\left\{\mathrm{e}^{i b \vec{n} \cdot \vec{\Lambda}}\right\}$. In this basis, time-reversal $T(W)$ of an operator is given by $T(W)=\Lambda_{y} \Sigma_{y} W^{*} \Lambda_{y} \Sigma_{y}$.

${ }^{20}$ In this Brief Report, we use $\hat{O}$ if operator $O$ is a $4 \times 4$ matrix in valley-sublattice space and $\mathscr{O}$ if $O$ is an $8 \times 8$ matrix in Keldyshvalley-sublattice space. Also, we suppress the unit matrix symbol everywhere in the text.

${ }^{21}$ B. L. Altshuler, JETP Lett. 41, 648 (1985) [Pis'ma Zh. Eksp. Teor. Fiz. 41, 530 (1985)]; P. A. Lee and A. D. Stone, Phys. Rev. Lett. 55, 1622 (1985); P. A. Lee et al., Phys. Rev. B 35, 1039 (1987).

${ }^{22}$ J. Rammer and H. Smith, Rev. Mod. Phys. 58, 323 (1986).

${ }^{23}$ Integration of Eq. (4) with respect to $\xi$ gives rise to terms of the form $i / \pi \int d \xi \xi G(\epsilon, \mathbf{n}(\epsilon+\xi), \mathbf{r}, \mathbf{t})=\Delta \epsilon g(\epsilon, \mathbf{n}, \mathbf{r}, t)$, where $\Delta \epsilon$ is a shift of the mass surface due to disorder and Fermi line warping. Such terms can be eliminated from the equation by recalculating the energy $\epsilon$ with respect to the shifted mass surface.

${ }^{24}$ Any matrix in the 4D isospin space can be expanded in the basis of linearly independent matrices $\left[(1+\Sigma \mathbf{n}),(1+\Sigma \mathbf{n}) \Sigma_{z}, \Sigma_{z}(1\right.$ $\left.+\Sigma \mathbf{n}), \Sigma_{z}(1+\Sigma \mathbf{n}) \Sigma_{z}\right]$. Substitution of the solution in form of Eq. (11) into the main order in Eq. (9) leaves only terms proportional to the last three matrices in the chosen basis in $\delta \hat{g}_{z}^{l}$.

${ }^{25}$ Conductivity of graphene monolayer with generic disorder is given by $\sigma=4 e^{2} \nu D_{0}=4 e^{2} \nu v^{2} \tau_{t r}^{0} / 2$ (where we took into account double spin degeneracy of carriers). Note that due to the valleyblock-diagonal form of the current operator $v_{x}=v \Sigma_{x}$ only $\tau_{t r}^{0}$ contributes to the conductance of graphene. At the same time, the variance of conductance contains contribution from mixed valley states and therefore depends on $\tau_{t r}^{l}, l=0, x, y, z$.

${ }^{26}$ B. Altshuler and D. Khmelnitski, JETP Lett. 42, 359 (1985).

${ }^{27}$ We take into account double spin degeneracy of carriers.

${ }^{28}$ This result suggests that the anomalously large variance of sample-to-sample conductance fluctuations observed in Ref. 15 is caused by a mechanism different from the phase interference which gives rise to the UCF.

${ }^{29}$ I. L. Aleiner and Y. M. Blanter, Phys. Rev. B 65, 115317 (2002).

${ }^{30}$ E. McCann and V. I. Falko, Phys. Rev. Lett. 96, 086805 (2006); J. Nilsson et al., Phys. Rev. B 73, 214418 (2006).

${ }^{31} \mathrm{M}$. Kharitonov and K. Efetov, arXiv:0801.0302 (unpublished) 